\title{
Duality of Moduli and Quasiconformal Mappings in Metric Spaces
}

https://doi.org/10.1515/agms-2020-0112

Received March 6, 2019; accepted June 5, 2020.

Abstract: We prove a duality relation for the moduli of the family of curves connecting two sets and the family of surfaces separating the sets, in the setting of a complete metric space equipped with a doubling measure and supporting a Poincaré inequality. Then we apply this to show that quasiconformal mappings can be characterized by the fact that they quasi-preserve the modulus of certain families of surfaces.

Keywords: quasiconformal mapping; modulus of a family of surfaces; finite perimeter; fine topology; Poincaré inequality

MSC: Primary: 30L10; Secondary: 26B30, 31E05

\section{Introduction}

A homeomorphism $f: X \rightarrow Y$ between two metric spaces $X, Y$ is said to be quasiconformal if there is a constant $H \geq 1$ such that for all $x \in X$,

$$
\limsup _{r \rightarrow 0^{+}} \frac{\sup _{y \in \bar{B}(x, r)} d_{Y}(f(x), f(y))}{\inf _{y \in X \backslash B(x, r)} d_{Y}(f(x), f(y))} \leq H .
$$

In metric measure spaces satisfying suitable conditions such as Ahlfors regularity and a Poincaré inequality, the study of quasiconformal mappings was begun by Heinonen and Koskela in [12] and by now the literature is extensive, see for example [3, 11, 13, 21, 25]. As in the classical Euclidean setting, there are also other notions of quasiconformality. For Ahlfors $Q$-regular spaces $X, Y$, a homeomorphism $f: X \rightarrow Y$ is said to be geometric quasiconformal if there is a constant $K \geq 1$ such that whenever $\Gamma$ is a family of curves in $X$, we have

$$
\frac{1}{K} \operatorname{Mod}_{Q}(f \Gamma) \leq \operatorname{Mod}_{Q}(\Gamma) \leq K \operatorname{Mod}_{Q}(f \Gamma) .
$$

For the definition of $Q$-modulus and all other concepts needed in the paper, we refer to Section 2. If both $X$ and $Y$ are complete and also support a $Q$-Poincare inequality, the two notions of quasiconformality are equivalent, see Theorem 9.8 in [13].

A fact that has received much less attention is that quasiconformal mappings also quasi-preserve the $\frac{Q}{Q-1}$-modulus of certain families of surfaces obtained as "essential boundaries" of sets of finite perimeter. This result was proved in Euclidean spaces by Kelly [18, Theorem 6.6]. In the metric space setting, the theory of functions of bounded variation (BV) and sets of finite perimeter was first developed by Ambrosio and Miranda $[2,23]$. The authors of the current paper together with Shanmugalingam extended Kelly's result to metric spaces in [15].

In the current paper, our main goal is to show that the converse holds as well: if a homeomorphism $f$ quasi-preserves the modulus of families of surfaces, then it is a quasiconformal mapping. Since the analogous

*Corresponding Author: Rebekah Jones, University of Cincinatti, Cincinatti, USA, E-mail: jones3rh@mail.uc.edu Panu Lahti, Universität Augsburg, Augsburg, Germany, E-mail: panu.lahti@math.ui-augsburg.de 
fact is already known to hold for families of curves, we invest most of our efforts in studying the duality of moduli of families of curves and surfaces. Specifically, for a nonempty bounded open set $\Omega \subset X$ and two disjoint sets $E, F \subset \Omega$, we consider the family of curves $\Gamma$ joining $E$ and $F$ in $\Omega$, and the family of surfaces $\mathcal{L}$ separating $E$ and $F$ in $\Omega$ in a suitable sense. Then we prove the following theorem; the precise formulation and assumptions on the sets $E$ and $F$ are given in Theorem 4.5.

Theorem 1.1. Let $1<p<\infty$ and suppose $X$ is a complete metric space equipped with a doubling measure and supporting a 1-Poincaré inequality. For some constant $C \geq 1$ depending only on $p$ and the space $X$, we have

$$
1 \leq \operatorname{Mod}_{\frac{p}{p-1}}(\mathcal{L})^{\frac{p-1}{p}} \operatorname{Mod}_{p}(\Gamma)^{\frac{1}{p}} \leq C .
$$

In Euclidean spaces, this was proved (with constant $C=1$ ) by Ziemer [26], and later by Aikawa and Ohtsuka who show in [1] that the same result holds for a more general weighted modulus with weights coming from the Muckenhoupt $A_{p}$-class. Combining Theorem 1.1 with the characterization of quasiconformal mappings by means of the moduli of curve families, we get the following theorem.

Theorem 1.2. Suppose that $X$ and $Y$ are complete Ahlfors $p$-regular metric spaces supporting a 1-Poincaré inequality. Suppose $f: X \rightarrow Y$ is a homeomorphism and there exists $C_{0}>0$ such that for every collection $\mathcal{L}$ of surfaces in $X$,

Then $f$ is quasiconformal.

$$
\operatorname{Mod}_{\frac{p}{p-1}}(f \mathcal{L}) \leq C_{0} \operatorname{Mod}_{\frac{p}{p-1}}(\mathcal{L})
$$

This is given, in a somewhat more general form, in Theorem 5.1. Results similar to Theorem 1.1 and Theorem 1.2 were very recently proved in the metric space setting by Lohvansuu and Rajala [22], but their viewpoint was somewhat different. In [22] (similarly to [26]) the authors understood a "surface" to be a set of finite codimension one Hausdorff measure separating $E$ and $F$ in a topological sense. By contrast, we understand surfaces to be sets of finite perimeter in the spirit of [18] and [15].

Moreover, we wish to study the problem under weaker assumptions: instead of Ahlfors regularity it is in fact enough to assume in Theorem 1.2 that the measures on $X$ and $Y$ are doubling and satisfy suitable one-sided growth bounds. Additionally, we do not assume the sets $E$ and $F$ to be closed, as was done in [22] and [26]. Working with more general sets makes it a rather subtle problem to find the correct definition for a "surface" that separates $E$ and $F$; for this we apply the concept of fine topology, relying on results proved in $[4,6,7]$. Hence our arguments combine the theory of quasiconformal mappings, BV theory, and fine potential theory in metric spaces.

\section{Notation and definitions}

In this section we gather the definitions and assumptions that we need in the paper.

Throughout the paper, $(X, d, \mu)$ is a complete metric measure space with $\mu$ a Radon measure. We assume that $X$ consists of at least 2 points. If a property holds outside a set with $\mu$-measure zero, we say that it holds almost everywhere, or a.e.

Given $x \in X$ and $r>0$, we denote an open ball by $B(x, r):=\{y \in X: d(y, x)<r\}$. Given that in a metric space a ball, as a set, could have more than one radius and more than one center, we will consider a ball to be also equipped with a radius and center; thus two different balls might correspond to the same set. We then denote $\operatorname{rad}(B):=r$ as the pre-assigned radius of the ball $B=B(x, r)$, and $a B:=B(x, a r)$ for $a>0$.

Definition 2.1. We say that $\mu$ is doubling if there exists a constant $C_{d} \geq 1$, called the doubling constant, such that

$$
0<\mu(2 B) \leq C_{d} \mu(B)<\infty
$$

for every ball $B$. 
We say that $(X, d, \mu)$ is Ahlfors Q-regular, with $Q>0$, if there is a constant $C_{A} \geq 1$ such that whenever $x \in X$ and $0<r<\operatorname{diam}(X)$, we have

$$
\frac{r^{Q}}{C_{A}} \leq \mu(B(x, r)) \leq C_{A} r^{Q} .
$$

Throughout the paper, we always assume $\mu$ to be doubling.

Definition 2.2. Let $A \subset X$. The codimension 1 Hausdorff measure of $A$ is given by

$$
\mathcal{H}(A):=\lim _{r \rightarrow 0^{+}} \inf \left\{\sum_{k \in I} \frac{\mu\left(B_{k}\right)}{\operatorname{rad}\left(B_{k}\right)} \mid A \subset \bigcup_{k \in I} B_{k} \text { where } \operatorname{rad}\left(B_{k}\right) \leq r \text { and } I \subset \mathbb{N}\right\} .
$$

Note that a complete metric space equipped with a doubling measure is always proper, that is, closed and bounded sets are compact. Given an open set $\Omega \subset X$, we write $u \in L_{\text {loc }}^{1}(\Omega)$ if $u \in L^{1}(V)$ for every open $V \Subset \Omega$; this expression means that $\bar{V}$ is a compact subset of $\Omega$. Other local spaces are defined analogously.

A curve is a continuous mapping from a compact interval into $X$, and a rectifiable curve is a curve with finite length. The length of a rectifiable curve $\gamma$ is denoted by $\ell_{\gamma}$. Every rectifiable curve can be parametrized by arc-length, see e.g. [10, Theorem 3.2]. In the following definitions, we let $1 \leq p<\infty$; in most of the paper we will assume that $1<p<\infty$.

Definition 2.3. Let $\mathcal{M}$ be a collection of Borel measures on $X$. The admissible class of $\mathcal{M}$, denoted $\mathcal{A}(\mathcal{M})$, is the set of all nonnegative Borel functions $\rho: X \rightarrow[0, \infty]$ such that

$$
\int_{X} \rho d \lambda \geq 1
$$

for all $\lambda \in \mathcal{M}$. The $p$-modulus of the family $\mathcal{M}$ is given by

$$
\operatorname{Mod}_{p}(\mathcal{M}):=\inf _{\rho \in \mathcal{A}(\mathcal{M})} \int_{X} \rho^{p} d \mu
$$

We say that a nonnegative Borel function $\rho$ is $p$-weakly admissible for the collection $\mathcal{M}$ if $\rho$ is admissible for all but a $p$-modulus zero collection of measures.

$\operatorname{Mod}_{p}$ is an outer measure on the class of all Borel measures, see [8]. There are two types of collections of measures associated with quasiconformal mappings. Firstly, given a collection $\Gamma$ of curves in $X$, we set $\Gamma$ to also denote the arc-length measures restricted to each curve in $\Gamma$; then the admissibility condition is replaced by

$$
\int_{\gamma} \rho d s \geq 1
$$

for every rectifiable $\gamma \in \Gamma$, where

$$
\int_{\gamma} \rho d s:=\int_{0}^{\ell_{\gamma}} \rho(\gamma(s)) d s
$$

for rectifiable $\gamma$. We say that a property holds for $p$-almost every curve if it fails only for a curve family with zero $p$-modulus. Secondly, for a collection $\mathcal{L}$ of sets of finite perimeter in a set $\Omega$, we consider the measures $P(U, \cdot)$ for each $U \in \mathcal{L}$ (see the definition given later).

Definition 2.4. Let $\Omega \subset X$ be $\mu$-measurable. Given a function $u: \Omega \rightarrow \overline{\mathbb{R}}$, a Borel function $g: \Omega \rightarrow[0, \infty]$ is said to be an upper gradient of $u$ in $\Omega$ if for every nonconstant rectifiable curve $\gamma$ in $\Omega$,

$$
|u(x)-u(y)| \leq \int_{\gamma} g d s,
$$


where $x$ and $y$ are the endpoints of $\gamma$. We interpret $|u(x)-u(y)|=\infty$ whenever either $|u(x)|$ or $|u(y)|$ is infinite. A function $u$ is said to be in the Newton-Sobolev class $N^{1, p}(\Omega)$ if $u \in L^{p}(\Omega)$ and there is an upper gradient $g$ of $u$ in $\Omega$ such that $g \in L^{p}(\Omega)$. We let

$$
\|u\|_{N^{1, p}(\Omega)}:=\|u\|_{L^{p}(\Omega)}+\inf \|g\|_{L^{p}(\Omega)},
$$

where the infimum is taken over upper gradients $g$ of $u$ in $\Omega$. We say that a nonnegative $\mu$-measurable function $g$ is a $p$-weak upper gradient of a function $u$ in $\Omega$ if (2.1) holds for $p$-almost every curve in $\Omega$.

If $u \in N_{\text {loc }}^{1, p}(\Omega)$, then there exists a minimal $p$-weak upper gradient of $u$ in $\Omega$, always denoted by $g_{u}$, satisfying $g_{u} \leq g$ a.e. in $\Omega$ for every $p$-weak upper gradient $g \in L_{\text {loc }}^{p}(\Omega)$ of $u$ in $\Omega$; see [4, Theorem 2.25] We refer the reader to $[4,14,24]$ for more details regarding mappings in $N_{\text {loc }}^{1, p}(\Omega)$.

Definition 2.5. We say that the space $X$ supports a $p$-Poincaré inequality if there exist constants $C_{P}>0$ and $\lambda \geq 1$ such that for all balls $B$ in $X$, all measurable functions $u$ on $X$ and all upper gradients $g$ of $u$,

$$
f_{B}\left|u-u_{B}\right| d \mu \leq C_{P} \operatorname{rad}(B)\left(f_{\lambda B} g^{p} d \mu\right)^{1 / p} .
$$

Here we denote the integral average of $u$ over $B$ by

$$
u_{B}:=f_{B} u d \mu:=\frac{1}{\mu(B)} \int_{B} u d \mu .
$$

We will assume throughout the paper that $X$ supports a 1-Poincaré inequality.

Definition 2.6. For any disjoint sets $E, F \subset X$, we define $\Gamma(E, F ; X)$ to be the collection of curves in $X$ joining $E$ and $F$. We say that $X$ is a Loewner space if there is a function $\phi:(0, \infty) \rightarrow(0, \infty)$ such that

$$
\operatorname{Mod}_{p}(\Gamma(E, F ; X)) \geq \phi(t)
$$

whenever $E$ and $F$ are two disjoint, nondegenerate continua (compact connected sets) such that

$$
t \geq \Delta(E, F):=\frac{\operatorname{dist}(E, F)}{\min \{\operatorname{diam}(E), \operatorname{diam}(F)\}} .
$$

Definition 2.7. The $p$-capacity of a set $A \subset X$ is given by

$$
\operatorname{Cap}_{p}(A):=\inf \|u\|_{N^{1, p}(X)},
$$

where the infimum is taken over functions $u \in N^{1, p}(X)$ satisfying $u \geq 1$ in $A$. If a property holds outside a set with $p$-capacity zero, we say that it holds $p$-quasieverywhere, or $p$-q.e.

We say that a set $V \subset X$ is $p$-quasiopen if for every $\varepsilon>0$ there is an open set $G \subset X$ such that $\operatorname{Cap}_{p}(G)<\varepsilon$ and $V \cup G$ is open.

The relative $p$-capacity of two sets $A \subset \Omega \subset X$ is given by

$$
\operatorname{cap}_{p}(A, \Omega):=\inf \int_{X} g_{u}^{p} d \mu
$$

where the infimum is over all functions $u \in N^{1, p}(X)$ such that $u \geq 1 p$-q.e. in $A$ and $u \leq 0$ in $X \backslash \Omega$. Recall that $g_{u}$ denotes the minimal $p$-weak upper gradient of $u$.

We know that $\operatorname{Cap}_{p}$ is an outer capacity in the following sense:

$$
\operatorname{Cap}_{p}(A)=\inf \left\{\operatorname{Cap}_{p}(W): W \supset A, W \text { is open }\right\}
$$

for any $A \subset X$, see e.g. [4, Theorem 5.31]. 
If $\Omega \subset X$ is $\mu$-measurable, then

$$
v=0 \text { p-q.e. in } \Omega \text { implies }\|v\|_{N^{1, p}(\Omega)}=0 \text {, }
$$

see [4, Proposition 1.61].

From now on, let $1<p<\infty$.

Definition 2.8. A set $E \subset X$ is $p$-thin at $x \in X$ if

$$
\int_{0}^{1}\left(\frac{\operatorname{cap}_{p}(E \cap B(x, t), B(x, 2 t))}{\operatorname{cap}_{p}(B(x, t), B(x, 2 t))}\right)^{\frac{1}{p-1}} \frac{d t}{t}<\infty .
$$

If $E$ is not $p$-thin at $x$, we say that it is $p$-thick. We denote the collection of all points where $E$ is $p$-thick by $b_{p} E$. If $X \backslash E$ is $p$-thin at each point $x \in E$, we say that the set $E$ is $p$-finely open. Then the $p$-fine topology on $X$ is the collection of all $p$-finely open sets.

Definition 2.9. Given a nonempty open set $\Omega$ and two disjoint sets $E, F$, we define the capacity of the condenser $(E, F ; \Omega)$ by

$$
\operatorname{cap}_{p}(E, F ; \Omega):=\inf \int_{\Omega} g_{u}^{p} d \mu,
$$

where the infimum is taken over all $u \in N^{1, p}(\Omega)$ satisfying $0 \leq u \leq 1$ in $\Omega, u=1$ in $E \cap \Omega$, and $u=0$ in $F \cap \Omega$.

Definition 2.10. A function $u \in N_{\text {loc }}^{1, p}(\Omega)$ is a $p$-minimizer in an open set $\Omega \subset X$ if for all $\varphi \in \operatorname{Lip}_{c}(\Omega)$ we have

$$
\int_{\{\varphi \neq 0\}} g_{u}^{p} d \mu \leq \int_{\{\varphi \neq 0\}} g_{u+\varphi}^{p} d \mu
$$

where $\operatorname{Lip}_{c}(\Omega)$ denotes the collection of Lipschitz functions that are compactly supported in $\Omega$. If the above inequality holds for all nonnegative $\varphi \in \operatorname{Lip}_{c}(\Omega)$, we say that $u$ is a $p$-superminimizer, and if it holds for all nonpositive $\varphi \in \operatorname{Lip}_{c}(\Omega)$, we say that $u$ is a $p$-subminimizer.

Next we consider the theory of BV functions in metric spaces.

Definition 2.11. For an open set $\Omega \subset X$ and $u \in L_{\text {loc }}^{1}(\Omega)$, the total variation of $u$ in $\Omega$ is given by

$$
\|D u\|(\Omega):=\inf \left\{\liminf _{n \rightarrow \infty} \int_{\Omega} g_{u_{n}} d \mu:\left(u_{n}\right)_{n \in \mathbb{N}} \subset \operatorname{Lip}_{\text {loc }}(\Omega), u_{n} \rightarrow u \operatorname{in} L_{\text {loc }}^{1}(\Omega)\right\} .
$$

We say $u \in L^{1}(\Omega)$ is of bounded variation on $\Omega$, denoted $u \in \operatorname{BV}(\Omega)$, if $\|D u\|(\Omega)<\infty$.

It is shown in [23, Theorem 3.4] that $\|D u\|$ is a Radon measure in $\Omega$ for any $u \in \operatorname{BV}_{\text {loc }}(\Omega)$. We call $\|D u\|$ the variation measure of $u$.

Definition 2.12. A measurable set $U \subset X$ has finite perimeter in $\Omega$ if $\left\|D \chi_{U}\right\|(\Omega)<\infty$. We call $\left\|D \chi_{U}\right\|$ the perimeter measure of $U$ and we will denote it $P(U, \cdot)$.

Definition 2.13. We say that $X$ supports a relative isoperimetric inequality if there exist constants $C_{I}>0$ and $\lambda \geq 1$ such that for all balls $B$ and for all measurable sets $U$, we have

$$
\min \{\mu(B \cap U), \mu(B \backslash U)\} \leq C_{I} \operatorname{rad}(B) P(U, \lambda B) .
$$

We know that when $\mu$ is doubling and $X$ supports a 1-Poincaré inequality, then it supports a relative isoperimetric inequality, see for example [20, Theorem 3.3] (in a slightly different form, this was proved earlier in [2, Theorem 4.3]). 
The noncentered Hardy-Littlewood maximal function of a function $\rho \in L_{\mathrm{loc}}^{1}(X)$ is defined by

$$
\mathcal{M} \rho(x):=\sup _{B \ni x} f_{B}|\rho| d \mu,
$$

where the supremum is taken over all open balls containing $x \in X$.

Finally we give the definition of quasiconformal mappings on metric spaces. Let $\left(Y, d_{Y}, \mu_{Y}\right)$ be another metric space equipped with a Radon measure $\mu_{Y}$.

Definition 2.14. For a function $f: X \rightarrow Y$, define for all $x \in X$ and $r>0$

$$
L_{f}(x, r):=\sup _{y \in \bar{B}(x, r)} d_{Y}(f(x), f(y)) \text { and } l_{f}(x, r):=\inf _{y \in X \backslash B(x, r)} d_{Y}(f(x), f(y)) .
$$

A homeomorphism $f$ is (metric) quasiconformal if there is a constant $H \geq 1$ such that for all $x \in X$ we have

$$
\limsup _{r \rightarrow 0^{+}} \frac{L_{f}(x, r)}{l_{f}(x, r)} \leq H .
$$

A homeomorphism $f$ is geometric quasiconformal if there is a constant $K \geq 1$ such that whenever $\Gamma$ is a family of curves in $X$, we have

$$
\frac{1}{K} \operatorname{Mod}_{p}(f \Gamma) \leq \operatorname{Mod}_{p}(\Gamma) \leq K \operatorname{Mod}_{p}(f \Gamma) .
$$

It is known that when both $X$ and $Y$ are Ahlfors $p$-regular and support a $p$-Poincaré inequality, the two notions of quasiconformality are equivalent, see Theorem 9.8 in [13]. We will make use of this fact in Section 5 , but we will give a self-contained proof where we only need somewhat weaker assumptions than Ahlfors regularity.

Standing assumptions: Throughout this paper we will assume that $1<p<\infty$ and that $(X, d, \mu)$ is a complete metric measure space that supports a 1-Poincare inequality, such that $\mu$ is doubling. We will use the letter $C$ to denote various nonnegative constants that depend only on $p$ and the space $X$, and the value of $C$ could differ at each occurrence.

\section{Background results}

In this section we will gather most of the background results needed in the paper. We start with the following coarea formula for BV functions, which is stated in Remark 4.3 of [23].

Theorem 3.1. Suppose $\Omega \subset X$ is open and $u \in \operatorname{BV}(\Omega)$. For each $t \in \mathbb{R}$, denote the super-level set of $u$ by $\{u>t\}:=\{x \in \Omega: u(x)>t\}$. Then for every nonnegative Borel function $\phi$ on $\Omega$ and every Borel set $A \subset \Omega$,

$$
\int_{\mathbb{R}}\left(\int_{A} \phi d P(\{u>t\}, \cdot)\right) d t=\int_{A} \phi d\|D u\| .
$$

We have the following "continuity from below" for families of measures; for a proof see Lemma 2.3 in [27].

Lemma 3.2. If $\left\{\mathcal{L}_{j}\right\}_{j \in \mathbb{N}}$ is a sequence of families of Borel measures such that $\mathcal{L}_{j} \subset \mathcal{L}_{j+1}$ for each $j$, then

$$
\operatorname{Mod}_{p}\left(\bigcup_{j \in \mathbb{N}} \mathcal{L}_{j}\right)=\lim _{j \rightarrow \infty} \operatorname{Mod}_{p}\left(\mathcal{L}_{j}\right)
$$

By applying Fuglede’s and Mazur's lemmas, see e.g. [13, p.19, p.131], we get the following. 
Lemma 3.3. Let $\mathcal{L}$ be a family of Borel measures with $\operatorname{Mod}_{p}(\mathcal{L})<\infty$. Then there exists a p-weakly admissible function $\rho$ such that

$$
\int_{X} \rho^{p} d \mu=\operatorname{Mod}_{p}(\mathcal{L}) .
$$

The following lemma is proved in [22, Lemma 5.2].

Lemma 3.4. If $\rho$ is a $p /(p-1)$-integrable, $p /(p-1)$-weakly admissible function for a family of Borel measures $\mathcal{L}$ such that

$$
\operatorname{Mod}_{\frac{p}{p-1}}(\mathcal{L})=\int_{X} \rho^{\frac{p}{p-1}} d \mu
$$

and $\phi$ is another $p /(p-1)$-integrable, $p /(p-1)$-weakly admissible function for $\mathcal{L}$, then

$$
\operatorname{Mod}_{\frac{p}{p-1}}(\mathcal{L}) \leq \int_{X} \phi \rho^{\frac{1}{p-1}} d \mu .
$$

We note that various results that we cite, such as the following theorem, rely on assuming the space to support a $p$-Poincaré inequality, but this follows via Hölder's inequality from the 1-Poincaré inequality that is our standing assumption.

Theorem 3.5. Suppose that $X$ satisfies the lower mass bound

$$
\mu(B(x, r)) \geq c r^{p}
$$

for all $x \in X$ and $0<r<\operatorname{diam}(X)$, and some constant $c>0$. Then $X$ is a Loewner space.

Proof. See Theorem 5.7 in [12]. Note that the so-called $\varphi$-convexity assumed in this theorem holds since under our assumptions the space is quasiconvex, meaning that every pair of points can be joined by a curve whose length is at most a constant number times the distance between the points; see e.g. [4, Theorem 4.32].

The space $X$ is linearly locally connected in the following sense.

Theorem 3.6. Suppose $X$ satisfies the upper mass bound

$$
\mu(B(x, r)) \leq C_{0} r^{p}
$$

for all $x \in X$ and $r>0$, and a constant $C_{0}>0$. Then there exists a constant $C_{1} \geq 1$ such that for every ball $B=B(x, r)$, any pair of points in $B \backslash \frac{1}{2} B$ can be joined by a curve in $B\left(x, C_{1} r\right) \backslash B\left(x, r / C_{1}\right)$.

Proof. See Remark 3.19 in [12]; note that there it is also assumed that the space is of Hausdorff dimension $p$, but this is not needed in the proof.

Finally we give a few results concerning superminimizers; recall Definition 2.10. Let $W \subset X$ be an open set. We define the lsc-regularization (lower semicontinuous regularization) of a function $u$ on $W$ by

$$
u^{*}(x):=\lim _{r \rightarrow 0} \underset{B(x, r)}{\operatorname{essinf}} u, \quad x \in W .
$$

The following proposition is given as part of Theorem 8.22 in [4].

Proposition 3.7. If $u$ is a p-superminimizer in $W$, then $u^{*}$ is lower semicontinuous in $W$ and $u=u^{*} p$-q.e. in $W$.

More precisely, the fact that $u=u^{*} p$-q.e. in $W$ is given in the proof of [4, Theorem 8.22]. By (2.2) we know that $u^{*}$ is still a $p$-superminimizer.

It is a well known fact that superharmonic functions are finely continuous; this was shown in the metric space setting in [7] and [19]. Here we record this result in the following theorem, which follows by combining Proposition 7.12, Theorem 9.24(a,c), and Theorem 11.38 of [4]. 
Theorem 3.8. Let $u$ be a $p$-superminimizer in $W$. Then $u^{*}$ is continuous with respect to the $p$-fine topology in $W$.

\section{Proof of Theorem 1.1}

We will consider the following families of curves and surfaces; recall the concept of capacitary thinness from Definition 2.8.

Definition 4.1. For an open set $\Omega \subset X$ and any disjoint sets $E, F \subset X$, we define $\Gamma(E, F ; \Omega)$ to be the collection of curves in $\Omega$ joining $E \cap \Omega$ and $F \cap \Omega$. We also define the collection of measures

$$
\begin{aligned}
\mathcal{L}(E, F ; \Omega):=\{P(U, \Omega \cap \cdot): U \subset X \text { is } \mu \text {-measurable with } \\
\left.b_{p} E \cap \Omega \subset \operatorname{int}(U) \text { and } b_{p} F \cap \Omega \subset \operatorname{ext}(U)\right\},
\end{aligned}
$$

where $\operatorname{ext}(U)=X \backslash \bar{U}$.

By an abuse of terminology, we will also talk about the sets $U$ belonging to $\mathcal{L}(E, F ; \Omega)$. Essentially, the boundaries of $U$ are "surfaces" that "separate" $E$ and $F$ in $\Omega$, but since we do not assume $E$ and $F$ to necessarily be compact subsets of $\Omega$, the choice of the correct definition for $\mathcal{L}(E, F ; \Omega)$ becomes rather subtle. If one would employ the usual definition where the surfaces need to stay at a strictly positive distance from $E$ and $F$, it would be difficult to prove the lower bound of Theorem 1.1. On the other hand, if one allows the surfaces to "touch" $E$ and $F$ significantly, then it becomes difficult to prove the upper bound. For this reason, we allow the surfaces to "touch" $E$ and $F$ only at capacitary thinness points.

Throughout this section, we will abbreviate $\mathcal{L}=\mathcal{L}(E, F ; \Omega)$ and $\Gamma=\Gamma(E, F ; \Omega)$. We begin by proving the lower bound.

Proposition 4.2. Let $\Omega \subset X$ be nonempty, open and bounded and let $E, F \subset X$ with $\bar{E} \cap \bar{F}=\varnothing$. Then $\operatorname{Mod}_{p}(\Gamma)<$ $\infty$, and if also $\operatorname{Mod}_{\frac{p}{p-1}}(\mathcal{L})<\infty$, then

$$
1 \leq \operatorname{Mod}_{\frac{p}{p-1}}(\mathcal{L})^{\frac{p-1}{p}} \operatorname{Mod}_{p}(\Gamma)^{\frac{1}{p}}
$$

Proof. Since $\bar{E} \cap \bar{\Omega}$ and $\bar{F} \cap \bar{\Omega}$ are two disjoint compact sets, we have $d:=\operatorname{dist}(\bar{E} \cap \bar{\Omega}, \bar{F} \cap \bar{\Omega})>0$ and so $\operatorname{Mod}_{p}(\Gamma)<\infty$; e.g. $d^{-1} \chi_{\Omega}$ is an admissible function. By [12, Proposition 2.17] and [16, Theorem 1.11] we have

$$
\operatorname{cap}_{p}(E, F ; \Omega)=\operatorname{Mod}_{p}(\Gamma) ;
$$

recall Definition 2.9. Then by [5, Theorem 5.13] we find a capacitary potential of $E$ and $F$ in $\Omega$, that is, a function $u \in N^{1, p}(\Omega)$ such that $0 \leq u \leq 1$ in $\Omega, u=1$ in $E \cap \Omega, u=0$ in $F \cap \Omega$, and

$$
\operatorname{cap}_{p}(E, F ; \Omega)=\int_{\Omega} g_{u}^{p} d \mu .
$$

We find two disjoint open sets $W_{1}, W_{2} \subset \Omega$ with $\bar{E} \cap \Omega \subset W_{1}$ and $\bar{F} \cap \Omega \subset W_{2}$. Since $u$ is a capacitary potential, for any nonnegative $\varphi \in \operatorname{Lip}_{c}\left(W_{1}\right) \subset \operatorname{Lip}_{c}(\Omega)$ we have that $u+\varphi$ is admissible for $\operatorname{cap}_{p}(E, F ; \Omega)$ and so

$$
\int_{\Omega} g_{u}^{p} d \mu \leq \int_{\Omega} g_{u+\varphi}^{p} d \mu,
$$

and so by the locality of minimal weak upper gradients (see e.g. [4, Corollary 2.21]),

$$
\int_{\{\varphi \neq 0\}} g_{u}^{p} d \mu \leq \int_{\{\varphi \neq 0\}} g_{u+\varphi}^{p} d \mu .
$$


Thus $u$ is superminimizer in $W_{1}$, and analogously a subminimizer in $W_{2}$. Let $u^{*}$ be the lsc-regularization of $u$ in $W_{1}$ and the analogously defined usc-regularization of $u$ in $W_{2}$, and $u^{*}=u$ in $\Omega \backslash\left(W_{1} \cup W_{2}\right)$. Then by Proposition 3.7, $u^{*}$ is lower semicontinuous in $W_{1}$ and, analogously, upper semicontinuous in $W_{2}$, and $u=u^{*}$ $p$-q.e. in $\Omega$.

Let $\mathcal{L}^{\dagger 7}$ be the collection of super-level sets of $u^{*}, U_{t}:=\left\{x \in \Omega: u^{*}(x)>t\right\}$, for $t \in(0,1)$. By Theorem 3.8 we have $u^{*}=1$ in $b_{p} E \cap \Omega$. Thus the sets $U_{t} \cap W_{1}$, for $t \in(0,1)$, are open and contain $b_{p} E \cap \Omega$, and so each set $\operatorname{int}\left(U_{t}\right)$ contains $b_{p} E \cap \Omega$. Analogously, $b_{p} F \cap \Omega \subset \operatorname{ext}\left(U_{t}\right)$ for all $t \in(0,1)$. In conclusion we have $\mathcal{L}^{円} \subset \mathcal{L}$, or more precisely $P\left(U_{t}, \Omega \cap \cdot\right)$ is in $\mathcal{L}$ for every $t \in(0,1)$. Thus $\operatorname{Mod}_{\frac{p}{p-1}}\left(\mathcal{L}^{円}\right) \leq \operatorname{Mod}_{\frac{p}{p-1}}(\mathcal{L})<\infty$.

Let $\rho \in L^{p /(p-1)}(X)$ be any admissible function for $\operatorname{Mod}_{\frac{p}{p-1}}\left(\mathcal{L}^{\dagger 7}\right)$. By e.g. [4, Proposition 2.44] we know that $g_{u, 1} \leq g_{u}$ in $\Omega$, where $g_{u, 1}$ and $g_{u}$ are the minimal 1-weak and $p$-weak upper gradients, respectively, of $u$ in $\Omega$. Thus also $u \in N^{1,1}(\Omega)$. Since $\operatorname{Lip}_{\text {loc }}(\Omega)$ is dense in $N^{1,1}(\Omega)$, see [4, Theorem 5.47], it follows that $u \in \operatorname{BV}(\Omega)$ with $d\|D u\| \leq g_{u, 1} d \mu \leq g_{u} d \mu$ in $\Omega$. Using also the coarea formula of Theorem 3.1, we get

$$
\begin{aligned}
1 & \leq \int_{0}^{1}\left(\int_{\Omega} \rho d P\left(U_{t}, \cdot\right)\right) d t \\
& =\int_{\Omega} \rho d\|D u\| \quad \| \text { since } u^{*}=u \text { a.e. } \\
& \leq \int_{\Omega} \rho g_{u} d \mu \\
& \leq\left(\int_{\Omega} \rho^{\frac{p}{p-1}} d \mu\right)^{\frac{p-1}{p}}\left(\int_{\Omega} g_{u}^{p} d \mu\right)^{\frac{1}{p}} \\
& =\left(\int_{\Omega} \rho^{\frac{p}{p-1}} d \mu\right)^{\frac{p-1}{p}} \operatorname{Mod}_{p}(\Gamma)^{\frac{1}{p}},
\end{aligned}
$$

using also (4.1). Taking the infimum over admissible $\rho$, we get

$$
1 \leq \operatorname{Mod}_{\frac{p}{p-1}}\left(\mathcal{L}^{\mp 1}\right)^{\frac{p-1}{p}} \operatorname{Mod}_{p}(\Gamma)^{\frac{1}{p}} \leq \operatorname{Mod}_{\frac{p}{p-1}}(\mathcal{L})^{\frac{p-1}{p}} \operatorname{Mod}_{p}(\Gamma)^{\frac{1}{p}} .
$$

In the case where $E$ and $F$ are compact, we get the lower bound also for the following smaller family of surfaces:

$$
\mathcal{L}^{*}:=\mathcal{L}^{*}(E, F ; \Omega):=\{P(U, \Omega \cap \cdot): E \subset \operatorname{int}(U) \text { and } F \subset \operatorname{ext}(U)\} .
$$

Proposition 4.3. Let $\Omega \subset X$ be a nonempty bounded domain and let $E, F \subset \Omega$ be disjoint nonempty compact sets. If $\operatorname{Mod}_{\frac{p}{p-1}}\left(\mathcal{L}^{*}\right)<\infty$, then

$$
1 \leq \operatorname{Mod}_{\frac{p}{p-1}}\left(\mathcal{L}^{*}\right)^{\frac{p-1}{p}} \operatorname{Mod}_{p}(\Gamma)^{\frac{1}{p}} .
$$

Proof. The proof is almost the same as for Proposition 4.2; we only need to note that since $E$ and $F$ are compact, according to Theorem 1.1 in [17] we find for every $\varepsilon>0$ a function $u \in \operatorname{Lip}_{\text {loc }}(\Omega)$ with $0 \leq u \leq 1$ in $\Omega$, $u=1$ in $E, u=0$ in $F$, and

$$
\int_{\Omega} g_{u}^{p} d \mu<\operatorname{cap}_{p}(E, F ; \Omega)+\varepsilon .
$$

Then we can consider the super-level sets $\{x \in \Omega: u(x)>t\}$ for $t \in(0,1)$, which all belong to $\mathcal{L}^{*}$.

Now we prove the upper bound. Part of the idea for the following proof came from Lohvansuu and Rajala [22]; the authors would like to thank them for sharing an early version of their manuscript. 
Proposition 4.4. Let $\Omega \subset X$ be open and $E, F \subset X$ be disjoint sets with $\operatorname{Cap}_{p}\left(b_{p} E \backslash E\right)=0$ and $\operatorname{Cap}_{p}\left(b_{p} F \backslash F\right)=$ 0. If $\operatorname{Mod}_{\frac{p}{p-1}}(\mathcal{L})=\infty$ then $\operatorname{Mod}_{p}(\Gamma)=0$, and if $0<\operatorname{Mod}_{\frac{p}{p-1}}(\mathcal{L})<\infty$ then

$$
\operatorname{Mod}_{\frac{p}{p-1}}(\mathcal{L})^{\frac{p-1}{p}} \operatorname{Mod}_{p}(\Gamma)^{\frac{1}{p}} \leq C
$$

for a constant $C$.

Note that in particular, a closed set $E$ satisfies $\operatorname{Cap}_{p}\left(b_{p} E \backslash E\right)=0$.

Proof. Let $x \in X \backslash b_{p} E$. Since $\operatorname{Cap}_{p}\left(b_{p} E \backslash E\right)=0$, by definition of the variational capacity we get

$$
\operatorname{cap}_{p}\left(B(x, t) \cap b_{p} E, B(x, 2 t)\right) \leq \operatorname{cap}_{p}(B(x, t) \cap E, B(x, 2 t))
$$

for every $t>0$. Thus

$$
\int_{0}^{1}\left(\frac{\operatorname{cap}_{p}\left(B(x, t) \cap b_{p} E, B(x, 2 t)\right)}{\operatorname{cap}_{p}(B(x, t), B(x, 2 t))}\right)^{\frac{1}{p-1}} \frac{d t}{t} \leq \int_{0}^{1}\left(\frac{\operatorname{cap}_{p}(B(x, t) \cap E, B(x, 2 t))}{\operatorname{cap}_{p}(B(x, t), B(x, 2 t))}\right)^{\frac{1}{p-1}} \frac{d t}{t}<\infty .
$$

Thus, $X \backslash b_{p} E$ is $p$-finely open. Similarly, $X \backslash b_{p} F$ is $p$-finely open and so $X \backslash\left(b_{p} E \cup b_{p} F\right)$ is as well.

Now by Theorem 1.4 in [6], we have that $X \backslash\left(b_{p} E \cup b_{p} F\right)$ is $p$-quasiopen. Then for each $i \in \mathbb{N}$, we can find an open set $G_{i} \subset X$ with $\operatorname{Cap}_{p}\left(G_{i}\right)<1 / i$ and so that $\left(X \backslash\left(b_{p} E \cup b_{p} F\right)\right) \cup G_{i}$ is open. We can assume that the sets $G_{i}$ form a decreasing sequence. Furthermore, we know that $\operatorname{Cap}_{p}\left(E \backslash b_{p} E\right)=0$ and $\operatorname{Cap}_{p}\left(F \backslash b_{p} F\right)=0$ (see Corollary 1.3 in [6]) so then since $\mathrm{Cap}_{p}$ is an outer capacity, we can choose $G_{i}$ to contain $E \Delta b_{p} E$ and $F \Delta b_{p} F$, where $\Delta$ denotes the symmetric difference. We now have that $\left(b_{p} E \cup b_{p} F\right) \backslash G_{i}=(E \cup F) \backslash G_{i}$ and this is a closed set.

Take open sets $\Omega_{1} \Subset \Omega_{2} \Subset \ldots \Subset \Omega$ with $\Omega=\cup_{i=1}^{\infty} \Omega_{i}$. Define

$$
\Gamma_{i}:=\left\{\gamma \in \Gamma(E, F ; \Omega):|\gamma| \subset \Omega_{i} \backslash G_{i}\right\},
$$

where $|\gamma|$ is the image of $\gamma$ in $X$. Fix $i \in \mathbb{N}$ and a rectifiable curve $\gamma \in \Gamma_{i}$ (assume for now that $\Gamma_{i} \neq \varnothing$ ). Let

$$
\mathcal{L}_{i, j}:=\left\{P(U, \Omega \cap \cdot): \operatorname{dist}\left(\overline{\Omega_{i}} \cap E \backslash G_{i}, X \backslash U\right)>j^{-1} \text { and } \operatorname{dist}\left(\overline{\Omega_{i}} \cap F \backslash G_{i}, U\right)>j^{-1}\right\} .
$$

Also fix $j \in \mathbb{N}$. We wish to construct an admissible function for $\mathcal{L}_{i, j}$. First we construct a Whitney covering of $\gamma$. Set

$$
\left.d(x):=\operatorname{dist}\left(x,\left(\overline{\Omega_{i}} \cap(E \cup F) \backslash G_{i}\right)\right) \cup(X \backslash \Omega)\right)
$$

and note that $d(x)>0$ for all $x \in|\gamma| \backslash(E \cup F)$. For $k \in \mathbb{Z}$ set

$$
|\gamma|_{k}:=\left\{x \in|\gamma|: 2^{k-1}<d(x) \leq 2^{k}\right\}
$$

and

$$
\mathcal{F}_{k}:=\left\{B\left(x, \frac{d(x)}{25 \lambda}\right): x \in|\gamma|_{k}\right\} .
$$

So $\mathcal{F}_{k}$ forms a cover of $|\gamma|_{k}$. Then by the 5-covering theorem, we can find a pairwise disjoint subcollection $\mathcal{G}_{k} \subset \mathcal{F}_{k}$ such that

$$
|\gamma|_{k} \subset \bigcup_{B \in \mathcal{F}_{k}} B \subset \bigcup_{B \in \mathcal{G}_{k}} 5 B .
$$

Since $|\gamma|_{k}$ is bounded, $\mathcal{G}_{k}$ is finite for each $k$. Letting $\mathcal{B}:=\bigcup_{k \in \mathbb{Z}} \mathcal{G}_{k}$, the collection of five times enlarged balls from $\mathcal{B}$ is a cover for $|\gamma| \backslash(E \cup F)$. Now for $U \in \mathcal{L}_{i, j}$, set

$$
T:=\sup \left\{t \in\left(0, \ell_{\gamma}\right): \frac{\mu(U \cap 5 B)}{\mu(5 B)} \geq \frac{1}{2} \text { for all } B \in \mathcal{B} \text { such that } \gamma(t) \in 5 B\right\} .
$$

We know that the above supremum is attained and $T \in\left(0, \ell_{\gamma}\right)$ since $\overline{\Omega_{i}} \cap b_{p} E \backslash G_{i}=\overline{\Omega_{i}} \cap E \backslash G_{i}$ is a compact subset of the open set $\operatorname{int}(U)$ and $\overline{\Omega_{i}} \cap b_{p} F \backslash G_{i}=\overline{\Omega_{i}} \cap F \backslash G_{i}$ is a compact subset of the open set ext $(U)$, and 
by the definition of $\mathcal{B}$. Let $B_{1} \in \mathcal{B}$ such that $\gamma(T) \in 5 B_{1}$. By continuity of $\gamma$ and definition of $T$, there exists $\kappa>0$ such that $\gamma(T-\kappa) \in 5 B_{1}$ and $\frac{\mu(U \cap 5 B)}{\mu(5 B)} \geq \frac{1}{2}$ for all $B \in \mathcal{B}$ such that $\gamma(T-\kappa) \in 5 B$. Thus $\frac{\mu\left(U \cap 5 B_{1}\right)}{\mu\left(5 B_{1}\right)} \geq \frac{1}{2}$. Again by continuity of $\gamma$, there exists $\delta>0$ such that $\gamma(T+\delta) \in 5 B_{1}$. Then since $T+\delta>T$, we know that there exists a ball $B_{2} \in \mathcal{B}$ with $\gamma(T+\delta) \in 5 B_{2}$ such that $\frac{\mu\left(U \cap 5 B_{2}\right)}{\mu\left(5 B_{2}\right)}<\frac{1}{2}$. So we have

$$
\frac{\mu\left(U \cap 5 B_{2}\right)}{\mu\left(5 B_{2}\right)}<\frac{1}{2} \leq \frac{\mu\left(U \cap 5 B_{1}\right)}{\mu\left(5 B_{1}\right)} .
$$

By the fact that $5 B_{1} \cap 5 B_{2}$ is nonempty (since it contains $\gamma(T+\delta)$ ), it is easy to check that $\operatorname{rad}\left(B_{1}\right) \leq$ $2 \operatorname{rad}\left(B_{2}\right) \leq 4 \operatorname{rad}\left(B_{1}\right)$. Hence $5 B_{2} \subset 25 B_{1}$. By using first (4.3) and the doubling property and then the relative isoperimetric inequality of Definition 2.13, we get for some constant $\widetilde{C}>0$ (depending only on the doubling constant)

$$
\frac{1}{\widetilde{C}} \leq \min \left\{\frac{\mu\left(25 B_{1} \cap U\right)}{\mu\left(25 B_{1}\right)}, \frac{\mu\left(25 B_{1} \backslash U\right)}{\mu\left(25 B_{1}\right)}\right\} \leq \frac{25 C_{I} \operatorname{rad}\left(B_{1}\right)}{\mu\left(B_{1}\right)} P\left(U, 25 \lambda B_{1}\right) .
$$

Choose $K_{j} \in \mathbb{Z}$ so that $2^{K_{j}}<\frac{1}{2} \min \left\{\frac{1}{j}, \operatorname{dist}\left(\Omega_{i}^{円}, X \backslash \Omega\right)\right\}$. Then for any $k \leq K_{j}$ and $B \in \mathcal{G}_{k}$, either $25 B \subset U$ or $25 B$ and $U$ are disjoint, which implies that $\frac{\mu(U \cap 25 B)}{\mu(25 B)} \in\{0,1\}$. Therefore we know that $B_{1} \in \mathcal{G}_{k}$ for some $k \geq K_{j}$. Define

$$
\phi_{i, j}:=25 C_{I} \widetilde{C} \sum_{k \geq K_{j}} \sum_{B \in \mathcal{G}_{k}} \frac{\operatorname{rad}(B)}{\mu(B)} \chi_{25 \lambda B} .
$$

Recall that $\mathcal{G}_{k}$ is finite for each $k$. Also $|\gamma|$ is bounded, so there exists a $K_{0} \in \mathbb{Z}$ such that $\mathcal{G}_{k}$ is empty for all $k \geq K_{0}$. Hence the function $\phi_{i, j}$ is $p /(p-1)$-integrable. Furthermore, $\phi_{i, j}$ is admissible for $\mathcal{L}_{i, j}$, since for any $U \in \mathcal{L}_{i, j}$, by (4.4) we have

$$
\begin{aligned}
\int_{\Omega} \phi_{i, j} d P(U, \cdot) & =25 C_{I} \widetilde{C} \int_{\Omega} \sum_{k \geq K_{j}} \sum_{B \in \mathcal{G}_{k}} \frac{\operatorname{rad}(B)}{\mu(B)} \chi_{25 \lambda B} d P(U, \cdot) \\
& =25 C_{I} \widetilde{C} \sum_{k \geq K_{j}} \sum_{B \in \mathcal{G}_{k}} \frac{\operatorname{rad}(B)}{\mu(B)} P(U, 25 \lambda B) \\
& \geq 1 .
\end{aligned}
$$

Using Lemma 3.3, pick a $p /(p-1)$-weakly admissible function $\rho_{i, j}$ such that

$$
\operatorname{Mod}_{\frac{p}{p-1}}\left(\mathcal{L}_{i, j}\right)=\int_{X} \rho_{i, j}^{\frac{p}{p-1}} d \mu .
$$

Recall the definition of the noncentered Hardy-Littlewood maximal function from (2.3). We now apply Lemma 3.4 which gives

$$
\begin{aligned}
\operatorname{Mod}_{\frac{p}{p-1}}\left(\mathcal{L}_{i, j}\right) & \leq \int_{X} \phi_{i, j} \rho_{i, j}^{\frac{1}{p-1}} d \mu \\
& \leq C \int_{X} \sum_{k \geq K_{j}} \sum_{B \in \mathcal{G}_{k}} \frac{\operatorname{rad}(B)}{\mu(B)} \chi_{25 \lambda B} \rho_{i, j}^{\frac{1}{p-1}} d \mu \\
& \leq C \sum_{k \in \mathbb{Z}} \sum_{B \in \mathcal{G}_{k}} \frac{\operatorname{rad}(B)}{\mu(B)} \int_{25 \lambda B} \rho_{i, j}^{\frac{1}{p-1}} d \mu \\
& \leq C \sum_{k \in \mathbb{Z}} \sum_{B \in \mathcal{G}_{k}} \operatorname{rad}(B) f_{25 \lambda B} \rho_{i, j}^{\frac{1}{p-1}} d \mu \\
& \leq C \sum_{k \in \mathbb{Z}} \sum_{B \in \mathcal{G}_{k}} \operatorname{rad}(B) \inf _{x \in B} \mathcal{M} \rho_{i, j}^{\frac{1}{p-1}}(x) \\
& \leq C \int_{\gamma} \mathcal{M} \rho_{i, j}^{\frac{1}{p-1}} d s ;
\end{aligned}
$$


the last inequality holds because the curve $\gamma$ travels at least the length $\operatorname{rad}(B)$ inside $B$, and the balls in each $\mathcal{G}_{k}$ are pairwise disjoint and clearly two balls $B_{1} \in \mathcal{G}_{k}$ and $B_{2} \in \mathcal{G}_{l}$ can only intersect if $|k-l|=1$.

Now we show that $\cup_{j} \mathcal{L}_{i, j}=\mathcal{L}$. First note that

$$
\bigcup_{j} \mathcal{L}_{i, j}=\left\{U \in \mathcal{L}: \operatorname{dist}\left(\overline{\Omega_{i}} \cap E \backslash G_{i}, X \backslash U\right)>0 \text { and } \operatorname{dist}\left(\overline{\Omega_{i}} \cap F \backslash G_{i}, U\right)>0\right\} .
$$

Now if we take $U \in \mathcal{L}$, then $\overline{\Omega_{i}} \cap b_{p} E \backslash G_{i} \subset \Omega \cap b_{p} E \subset \operatorname{int}(U)$, i.e. $\overline{\Omega_{i}} \cap b_{p} E \backslash G_{i}$ is a compact subset of the open set $\operatorname{int}(U)$. Hence there is a strictly positive distance between $\overline{\Omega_{i}} \cap b_{p} E \backslash G_{i}=\overline{\Omega_{i}} \cap E \backslash G_{i}$ and $X \backslash U$. A similar argument shows that there is a strictly positive distance between $\overline{\Omega_{i}} \cap F \backslash G_{i}$ and $U$. Therefore $U \in \cup_{j} \mathcal{L}_{i, j}$, proving that $\cup_{j} \mathcal{L}_{i, j}=\mathcal{L}$.

Now note that since we are assuming $\operatorname{Mod}_{\frac{p}{p-1}}(\mathcal{L}) \neq 0$, and the families $\mathcal{L}_{i, j}$ are increasing in $j$, by Lemma 3.2 we have $\operatorname{Mod}_{\frac{p}{p-1}}\left(\mathcal{L}_{i, j}\right)>0$ for all sufficiently large $j \in \mathbb{N}$ (with $i$ still fixed). Thus by (4.5), $C \operatorname{Mod}_{\frac{p}{p-1}}\left(\mathcal{L}_{i, j}\right)^{-1} \mathcal{M} \rho_{i, j}^{\frac{1}{p-1}}$ is admissible for $\Gamma_{i}$. Therefore

$$
\operatorname{Mod}_{p}\left(\Gamma_{i}\right) \leq C \operatorname{Mod}_{\frac{p}{p-1}}\left(\mathcal{L}_{i, j}\right)^{-p} \int_{X}\left(\mathcal{M} \rho_{i, j}^{\frac{1}{p-1}}\right)^{p} d \mu
$$

Since the maximal function is a bounded operator from $L^{p}(X)$ to $L^{p}(X)$ when $1<p<\infty$, see e.g. [4, Theorem 3.13], we get

$$
\begin{aligned}
\operatorname{Mod}_{p}\left(\Gamma_{i}\right)^{\frac{1}{p}} & \leq C \operatorname{Mod}_{\frac{p}{p-1}}\left(\mathcal{L}_{i, j}\right)^{-1}\left\|\mathcal{M} \rho_{i, j}^{\frac{1}{p-1}}\right\|_{L^{p}(X)} \\
& \leq C \operatorname{Mod}_{\frac{p}{p-1}}\left(\mathcal{L}_{i, j}\right)^{-1}\left\|\rho_{i, j}^{\frac{1}{p-1}}\right\|_{L^{p}(X)} \\
& =C \operatorname{Mod}_{\frac{p}{p-1}}\left(\mathcal{L}_{i, j}\right)^{-1}\left(\int_{X} \rho_{i, j}^{\frac{p}{p-1}} d \mu\right)^{\frac{1}{p}} \\
& =C \operatorname{Mod}_{\frac{p}{p-1}}\left(\mathcal{L}_{i, j}\right)^{\frac{1-p}{p}} .
\end{aligned}
$$

Recalling that $\lim _{j \rightarrow \infty} \operatorname{Mod}_{\frac{p}{p-1}}\left(\mathcal{L}_{i, j}\right)=\operatorname{Mod}_{\frac{p}{p-1}}(\mathcal{L})$, we get

$$
\operatorname{Mod}_{p}\left(\Gamma_{i}\right)^{\frac{1}{p}} \leq C \operatorname{Mod}_{\frac{p}{p-1}}(\mathcal{L})^{\frac{1-p}{p}},
$$

and in particular $\operatorname{Mod}_{p}\left(\Gamma_{i}\right)=0$ if $\operatorname{Mod}_{\frac{p}{p-1}}(\mathcal{L})=\infty$. Note that (4.6) holds also if $\Gamma_{i}=\varnothing$.

Finally note that the sequence $\Gamma_{i}$ is increasing with $\bigcup_{i \in \mathbb{N}} \Gamma_{i}=\Gamma \backslash N$, where

$$
N:=\left\{\gamma \in \Gamma:|\gamma| \cap \bigcap_{i} G_{i} \neq \varnothing\right\} .
$$

But $\operatorname{Cap}_{p}\left(\cap_{i} G_{i}\right)=0$, and so $\operatorname{Mod}_{p}(N)=0$, see e.g. [4, Proposition 1.48]. So by Lemma 3.2 we have $\lim _{i \rightarrow \infty} \operatorname{Mod}_{p}\left(\Gamma_{i}\right)=\operatorname{Mod}_{p}(\Gamma \backslash N)=\operatorname{Mod}_{p}(\Gamma)$. Combining this with (4.6) above, we get

$$
\operatorname{Mod}_{p}(\Gamma)^{\frac{1}{p}}=\lim _{i \rightarrow \infty} \operatorname{Mod}_{p}\left(\Gamma_{i}\right)^{\frac{1}{p}} \leq C \operatorname{Mod}_{\frac{p}{p-1}}(\mathcal{L})^{\frac{1-p}{p}},
$$

and in particular $\operatorname{Mod}_{p}(\Gamma)=0$ if $\operatorname{Mod}_{\frac{p}{p-1}}(\mathcal{L})=\infty$.

Now Theorem 1.1 from the introduction follows from Proposition 4.2 and Proposition 4.4. We give the theorem in the following more precise form.

Theorem 4.5. Let $\Omega \subset X$ be nonempty, open and bounded and let $E, F \subset X$ be such that $\bar{E} \cap \bar{F}=\varnothing$ and $\operatorname{Cap}_{p}\left(b_{p} E \backslash E\right)=0$ and $\operatorname{Cap}_{p}\left(b_{p} F \backslash F\right)=0$. If $\operatorname{Mod}_{\frac{p}{p-1}}(\mathcal{L})=\infty$ then $\operatorname{Mod}_{p}(\Gamma)=0$, and else

$$
1 \leq \operatorname{Mod}_{\frac{p}{p-1}}(\mathcal{L})^{\frac{p-1}{p}} \operatorname{Mod}_{p}(\Gamma)^{\frac{1}{p}} \leq C
$$

for some constant $C \geq 1$. 


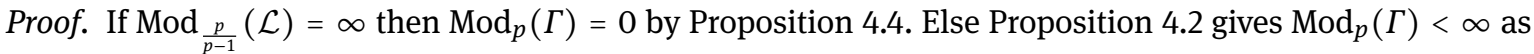
well as the lower bound of the theorem, and in particular this guarantees $0<\operatorname{Mod}_{p}(\Gamma)<\infty$. Then the upper bound follows from Proposition 4.4 .

\section{Proof of Theorem 1.2}

Now we can prove Theorem 1.2 given in the introduction. We give it in the following somewhat more general form.

Theorem 5.1. Suppose that $\left(Y, d_{Y}, \mu_{Y}\right)$ is another complete metric space that supports a 1-Poincaré inequality, such that $\mu_{Y}$ is doubling and

$$
\mu(B(x, r)) \geq C_{0}^{-1} r^{p} \quad \text { and } \quad \mu_{Y}(B(y, r)) \leq C_{0} r^{p}
$$

for all $x \in X, y \in Y, r>0$, and a constant $C_{0}>0$. Suppose $f: X \rightarrow Y$ is a homeomorphism such that for every collection of surfaces $\mathcal{L}=\mathcal{L}(E, F ; \Omega)$ with $\Omega \subset X$ nonempty, open and bounded and $E, F \subset \Omega$ compact, we have

$$
\operatorname{Mod}_{\frac{p}{p-1}}(f \mathcal{L}) \leq C_{0} \operatorname{Mod}_{\frac{p}{p-1}}(\mathcal{L})
$$

where

$$
f \mathcal{L}=\left\{P(f(U), f(\Omega) \cap \cdot): b_{p} E \cap \Omega \subset \operatorname{int}(U) \text { and } b_{p} F \cap \Omega \subset \operatorname{ext}(U)\right\} .
$$

Then $f$ is quasiconformal with a constant depending only on $C_{0}$, $p$, and the space $X$.

Of course, (5.1) is satisfied in particular if $X$ and $Y$ are both Ahlfors $p$-regular; recall Definition 2.1. Also recall the definitions of $L_{f}$ and $l_{f}$ from Definition 2.14 .

Proof. As complete metric spaces equipped with a doubling measure and supporting a Poincaré inequality, $X$ and $Y$ are quasiconvex, see e.g. [4, Theorem 4.32], and so for each of them a biLipschitz change in the metric gives a geodesic space (see Section 4.7 in [4]). Since the theorem is easily seen to be invariant under biLipschitz changes in the metrics, we can assume that $X$ and $Y$ are geodesic.

We want to apply Proposition 4.3 and Proposition 4.4 to suitable sets defined via the homeomorphism $f$. Fix $x \in X$ and $r>0$ and let $L:=L_{f}(x, r)$ and $l:=l_{f}(x, r)>0$. Suppose also that $L>2 C_{1} l$, where $C_{1}$ is the constant from Theorem 3.6. By choosing $r$ sufficiently small, we have $L<\operatorname{diam}(Y) / 4$ (we can assume that $\operatorname{diam}(Y)>0)$. Since $f(\bar{B}(x, r))$ is compact, there exists $y \in f(\bar{B}(x, r))$ such that $d_{Y}(f(x), y)=L$.

Let $E:=f^{-1}(\bar{B}(f(x), l))$, and $F:=f^{-1}\left(F_{*}\right)$ where $F_{*}$ is the maximal connected set containing $y$ and contained in $\bar{B}(f(x), M) \backslash B\left(f(x), L / C_{1}\right)$, for some fixed $M \geq 2 C_{1} L$. By Theorem 3.6 (note that here we use the upper bound in (5.1)) we have

$$
F_{*} \supset B\left(f(x), M / C_{1}\right) \backslash B(f(x), L) .
$$

Note that balls are connected in geodesic spaces, and $f$ is a homeomorphism, so $E$ and $F$ are connected. Both $E$ and $F$ are moreover closed, and since $X$ and $Y$ are proper, $f$ and $f^{-1}$ map bounded sets to bounded sets, and so $E$ and $F$ are also bounded and thus compact. Since $Y$ is connected, the set $F_{\star}$ and thus also the set $F$ consists of at least 2 points and so $\operatorname{diam}(F)>0$. If $r \rightarrow 0$ then $\operatorname{diam}(E) \rightarrow 0$, and thus by choosing $r$ even smaller if necessary, we can assume that $\operatorname{diam}(E)$ is less than $\operatorname{diam}(F)$. Note that $\Omega:=f^{-1}(B(f(x), M+1))$ is also bounded. Let $\Gamma=\Gamma(E, F ; \Omega)$ and $\mathcal{L}=\mathcal{L}(E, F ; \Omega)$.

For the family $f \Gamma$ consisting of the curves $f \circ \gamma$, with $\gamma \in \Gamma$, we have

$$
f \Gamma=\Gamma\left(\bar{B}(f(x), l), F_{*} ; B(f(x), M+1)\right) .
$$

From this it is easy to see that every curve in $\Gamma(E, F ; X)$ has a subcurve in $\Gamma$, and $\operatorname{so} \operatorname{Mod}_{p}(\Gamma)=$ $\operatorname{Mod}_{p}(\Gamma(E, F ; X))$; see e.g. [4, Lemma 1.34(c)]. Notice that $f^{-1}(y) \in F \cap \bar{B}(x, r)$, and we know that $x \in E$, 
so $\operatorname{dist}(E, F) \leq r$. It is straightforward to show that there is some $z \in X \backslash B(x, r)$ with $d_{Y}(f(x), f(z))=l$. Thus $r \leq \operatorname{diam}(E)$, which we noted to be less than $\operatorname{diam}(F)$, and so

$$
\frac{\operatorname{dist}(E, F)}{\min \{\operatorname{diam}(E), \operatorname{diam}(F)\}} \leq \frac{r}{r}=1 .
$$

By Theorem 3.5 we know that $X$ is a Loewner space (note that here we need the lower mass bound in (5.1)), and so

$$
\operatorname{Mod}_{p}(\Gamma)=\operatorname{Mod}_{p}(\Gamma(E, F ; X)) \geq \phi(1)>0,
$$

where $\phi$ is the Loewner function for $X$. We observe that every curve in $f \Gamma$ has a subcurve in the family $\Gamma\left(\bar{B}(f(x), l), Y \backslash B\left(f(x), L / C_{1}\right) ; Y\right)$. Thus

$$
\operatorname{Mod}_{p}(f \Gamma) \leq \operatorname{Mod}_{p}\left(\Gamma\left(\bar{B}(f(x), l), Y \backslash B\left(f(x), L / C_{1}\right)\right) ; Y\right) .
$$

Now by Proposition 5.3.9 in [14] we have

$$
\operatorname{Mod}_{p}(f \Gamma) \leq C_{2}\left(\log \frac{L}{C_{1} l}\right)^{1-p}
$$

for a constant $C_{2}$ depending only on $C_{0}$ and $p$ (here we need the upper mass bound in (5.1)).

Since $L<\operatorname{diam}(Y) / 4$ and $Y$ is connected, by (5.2) there exists a ball $B \subset F_{\star}$ with $\operatorname{rad}(B)=L / 2$. Then from the relative isoperimetric inequality of Definition 2.13 and the doubling property of $\mu_{Y}$ we see that for every $U \in f \mathcal{L}$,

$$
P(U, B(f(x), 2 \lambda M)) \geq\left(2 C_{I} M\right)^{-1} \min \left\{\mu_{Y}(B(f(x), l)), \mu_{Y}(B)\right\}=: c>0 .
$$

It follows that $\operatorname{Mod}_{p /(p-1)}(f \mathcal{L})<\infty$, as $c^{-1} \chi_{B(f(x), 2 \lambda M)}$ is an admissible test function. Recall the definition of the family $\mathcal{L}^{*} \subset \mathcal{L}$ from (4.2). For this family it is easy to verify (since $f$ is a homeomorphism) that

$$
f \mathcal{L}^{*}=\mathcal{L}^{*}\left(\bar{B}(f(x), l), F^{*} ; B(f(x), M+1)\right) .
$$

Thus by Proposition 4.3 and the assumption of the quasi-preservation of modulus of surfaces,

$$
1 \leq \operatorname{Mod}_{\frac{p}{p-1}}\left(f \mathcal{L}^{*}\right)^{\frac{p-1}{p}} \operatorname{Mod}_{p}(f \Gamma)^{\frac{1}{p}} \leq C_{0}^{\frac{p-1}{p}} \operatorname{Mod}_{\frac{p}{p-1}}(\mathcal{L})^{\frac{p-1}{p}} \operatorname{Mod}_{p}(f \Gamma)^{\frac{1}{p}} .
$$

Therefore, by Proposition 4.4 and since we $\operatorname{had} \operatorname{Mod}_{p}(\Gamma) \neq 0(\operatorname{recall}(5.3))$

$$
\operatorname{Mod}_{p}(\Gamma)^{\frac{1}{p}} \leq C_{0}^{\frac{p-1}{p}} \operatorname{Mod}_{p}(\Gamma)^{\frac{1}{p}} \operatorname{Mod}_{\frac{p}{p-1}}(\mathcal{L})^{\frac{p-1}{p}} \operatorname{Mod}_{p}(f \Gamma)^{\frac{1}{p}} \leq C_{0}^{\frac{p-1}{p}} C \operatorname{Mod}_{p}(f \Gamma)^{\frac{1}{p}} .
$$

Combining this with (5.3) and (5.4), we have

$$
\phi(1) \leq \operatorname{Mod}_{p}(\Gamma) \leq C_{0}^{p-1} C^{p} C_{2}\left(\log \frac{L}{C_{1} l}\right)^{1-p} .
$$

Thus

$$
\frac{L}{l} \leq C_{1} \exp \left(C_{0} C^{\frac{p}{p-1}} C_{2}^{\frac{1}{p-1}} \phi(1)^{\frac{1}{1-p}}\right) .
$$

Recall that we were assuming $L>2 C_{1} l$; in conclusion

$$
\limsup _{r \rightarrow 0^{+}} \frac{L_{f}(x, r)}{l_{f}(x, r)} \leq C_{1} \max \left\{2, \exp \left(C_{0} C^{\frac{p}{p-1}} C_{2}^{\frac{1}{p-1}} \phi(1)^{\frac{1}{1-p}}\right)\right\}
$$

for every $x \in X$. Therefore $f$ is quasiconformal.

Remark 5.2. Note that in the above proof we employed the family $\mathcal{L}^{*}$ because it is not clear that

$$
f \mathcal{L}=\mathcal{L}\left(\bar{B}(f(x), l), F_{\star} ; B(f(x), M+1)\right) .
$$

This is the case because it is not clear that

$$
b_{p} E \subset \operatorname{int}\left(f^{-1}(U)\right) \quad \text { and } \quad b_{p} F \subset \operatorname{ext}\left(f^{-1}(U)\right)
$$


for every $U \in \mathcal{L}\left(\bar{B}(f(x), l), F_{*} ; B(f(x), M+1)\right)$, as would be required in the definition of $\mathcal{L}(E, F ; \Omega)$. In other words, the image under $f$ or $f^{-1}$ of every "separating surface" might not be a "separating surface". It is known, at least in Ahlfors regular spaces, that a quasiconformal mapping (whose inverse is also quasiconformal) preserves the measure-theoretic interior, exterior, and boundary, see [9], [21, Theorem 6.1], and [15, Lemma 4.8]. If we knew a similar property to hold for capacitary thickness points, then the above problem would not arise. Thus we ask:

- If $f: X \rightarrow Y$ is a quasiconformal mapping, do we have $f\left(b_{p} E\right)=b_{p} f(E)$ for every (closed) set $E \subset X$ ?

Acknowledgement: The authors are grateful to Nageswari Shanmugalingam for advice and many discussions on the topic of the paper. The authors also thank Kai Rajala and the anonymous referee for feedback. The second author wishes to acknowledge the hospitality of the University of Cincinnati, where most of the research for this paper was conducted. The research was partially funded by the National Science Foundation (U.S.A.) grants DMS \#1500440 and DMS \#1800161.

\section{References}

[1] H. Aikawa and M. Ohtsuka, Extremal length of vector measures, Ann. Acad. Sci. Fenn. Math. 24 (1999), no. 1, 61-88.

[2] L. Ambrosio, Fine properties of sets of finite perimeter in doubling metric measure spaces, Calculus of variations, nonsmooth analysis and related topics. Set-Valued Anal. 10 (2002), no. 2-3, 111-128.

[3] C. Bishop, H. Hakobyan, and M. Williams, Quasisymmetric dimension distortion of Ahlfors regular subsets of a metric space, Geom. Funct. Anal. 26 (2016), no. 2, 379-421.

[4] A. Björn and J. Björn, Nonlinear potential theory on metric spaces, EMS Tracts in Mathematics, 17. European Mathematical Society (EMS), Zürich, 2011, xii+403 pp.

[5] A. Björn and J. Björn, Obstacle and Dirichlet problems on arbitrary nonopen sets in metric spaces, and fine topology, Rev. Mat. Iberoam. 31 (2015), no. 1, 161-214.

[6] A. Björn, J. Björn, and V. Latvala, The Cartan, Choquet and Kellogg properties for the fine topology on metric spaces, J. Anal. Math. 135 (2018), no. 1, 59-83.

[7] J. Björn, Fine continuity on metric spaces, Manuscripta Math. 125 (2008), no. 3, 369-381.

[8] B. Fuglede, Extremal length and functional completion, Acta Math. 98 (1957), 171-219.

[9] F. Gehring and J. C. Kelly, Quasi-conformal mappings and Lebesgue density, Discontinuous groups and Riemann surfaces (Proc. Conf., Univ. Maryland, College Park, Md., 1973), Ann. of Math. Studies 79, Princeton Univ. Press, Princeton, N.J., 1974, pp. 171-179.

[10] P. Hajtasz, Sobolev spaces on metric-measure spaces, Heat kernels and analysis on manifolds, graphs, and metric spaces (Paris, 2002), 173-218, Contemp. Math., 338, Amer. Math. Soc., Providence, RI, 2003.

[11] J. Heinonen and P. Koskela, Definitions of quasiconformality, Invent. Math. 120 (1995), no. 1, 61-79.

[12] J. Heinonen and P. Koskela, Quasiconformal maps in metric spaces with controlled geometry, Acta Math. 181 (1998), no. $1,1-61$.

[13] J. Heinonen, P. Koskela, N. Shanmugalingam, and J. Tyson, Sobolev classes of Banach space-valued functions and quasiconformal mappings, J. Anal. Math. 85 (2001), 87-139.

[14] J. Heinonen, P. Koskela, N. Shanmugalingam, and J. Tyson, Sobolev spaces on metric measure spaces: An approach based on upper gradients, New Mathematical Monographs, 27. Cambridge University Press, Cambridge, 2015, xii+434 pp.

[15] R. Jones, P. Lahti, and N. Shanmugalingam, Modulus of families of sets of finite perimeter and quasiconformal maps between metric spaces of globally Q-bounded geometry, to appear in Indiana University Mathematics Journal. https://arxiv.org/abs/1806.06211

[16] E. Järvenpää, M. Järvenpää, K. Rogovin, S. Rogovin, and N. Shanmugalingam, Measurability of equivalence classes and $M E C_{p}$-property in metric spaces, Rev. Mat. Iberoam. 23 (2007), no. 3, 811-830.

[17] S. Kallunki and N. Shanmugalingam, Modulus and continuous capacity, Ann. Acad. Sci. Fenn. Math. 26 (2001), no. 2 , 455-464.

[18] J. C. Kelly, Quasiconformal mappings and sets of finite perimeter, Trans. Amer. Math. Soc. 180 (1973), 367-387.

[19] R. Korte, A Caccioppoli estimate and fine continuity for superminimizers on metric spaces, Ann. Acad. Sci. Fenn. Math. 33 (2008), no. 2, 597-604.

[20] R. Korte and P. Lahti, Relative isoperimetric inequalities and sufficient conditions for finite perimeter on metric spaces, Ann. Inst. H. Poincaré Anal. Non Linéaire 31 (2014), no. 1, 129-154. 
[21] R. Korte, N. Marola, and N. Shanmugalingam, Quasiconformality, homeomorphisms between metric measure spaces preserving quasiminimizers, and uniform density property, Ark. Mat. 50 (2012), 111-134.

[22] A. Lohvansuu and K. Rajala, Duality of moduli in regular metric spaces, preprint 2018.

[23] M. Miranda, Jr., Functions of bounded variation on "good" metric spaces, J. Math. Pures Appl. (9) 82 (2003), no. 8, 9751004.

[24] N. Shanmugalingam, Newtonian spaces: An extension of Sobolev spaces to metric measure spaces, Rev. Mat. Iberoamericana 16(2) (2000), 243-279.

[25] M. Williams, Geometric and analytic quasiconformality in metric measure spaces, Proc. Amer. Math. Soc. 140 (2012), no. 4, 1251-1266.

[26] W. Ziemer, Extremal length and conformal capacity, Trans. Amer. Math. Soc. 126 1967 460-473.

[27] W. Ziemer, Extremal length and p-capacity, Michigan Math. J. 161969 43-51. 